\title{
Article
}

\section{TP53 Targeted Deep Sequencing of Cell-Free DNA in Esophageal Squamous Cell Carcinoma Using Low-Quality Serum: Concordance with Tumor Mutation}

\author{
Dariush Nasrollahzadeh 1,2, Gholamreza Roshandel ${ }^{3}$, Tiffany Myriam Delhomme ${ }^{2,4} @$, Patrice Hodonou Avogbe ${ }^{2}$,

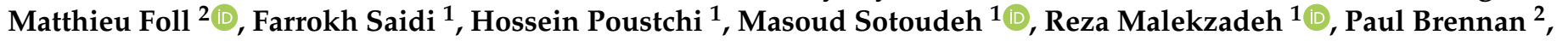 \\ James Mckay ${ }^{2}$, Pierre Hainaut ${ }^{5}$ and Behnoush Abedi-Ardekani ${ }^{2, *}$ (i)
}

1 Digestive Oncology Research Center, Digestive Disease Research Institute, Shariati Hospital, Tehran University of Medical Sciences, Tehran 14117-13135, Iran; neshelid@fellows.iarc.fr (D.N.); saidif@ams.ac.ir (F.S.); h.poustch@gmail.com (H.P.); masoud.sotoudeh.md@gmail.com (M.S.); dr.reza.malekzadeh@gmail.com (R.M.)

2 Genomic Epidemiology Branch, International Agency for Research on Cancer/World Health Organization (IARC/WHO), 69000 Lyon, France; tiffany.delhomme@irbbarcelona.org (T.M.D.); patrice.avogbe@gmail.com (P.H.A.); follM@iarc.fr (M.F.); brennanp@iarc.fr (P.B.); mckayj@iarc.fr (J.M.)

3 Golestan Research Center of Gastroenterology and Hepatology, Golestan University of Medical Sciences, Gorgan 49177-44563, Iran; drroshandel@goums.ac.ir

check for updates

Citation: Nasrollahzadeh, D.; Roshandel, G.; Delhomme, T.M.; Avogbe, P.H.; Foll, M.; Saidi, F.; Poustchi, H.; Sotoudeh, M.; Malekzadeh, R.; Brennan, P.; et al. TP53 Targeted Deep Sequencing of Cell-Free DNA in Esophageal Squamous Cell Carcinoma Using Low-Quality Serum: Concordance with Tumor Mutation. Int. J. Mol. Sci. 2021, 22, 5627. https://doi.org/ $10.3390 /$ ijms 22115627

Academic Editors: Ines Gockel and Asfar S. Azmi

Received: 7 April 2021

Accepted: 17 May 2021

Published: 26 May 2021

Publisher's Note: MDPI stays neutral with regard to jurisdictional claims in published maps and institutional affiliations.

Copyright: (c) 2021 by the authors. Licensee MDPI, Basel, Switzerland. This article is an open access article distributed under the terms and conditions of the Creative Commons Attribution (CC BY) license (https:/ / creativecommons.org/licenses/by/ $4.0 /)$.
4 Institute for Research in Biomedicine (IRB Barcelona), Barcelona Institute of Science and Technology, 08036 Barcelona, Spain

5 Institute for Advanced Biosciences, Inserm 1209 CNRS 5309 UGA, 38700 Grenoble, France; pierre.hainaut@univ-grenoble-alpes.fr

* Correspondence: abedib@iarc.fr

\begin{abstract}
Circulating cell-free DNA (cfDNA) is emerging as a potential tumor biomarker. CfDNAbased biomarkers may be applicable in tumors without an available non-invasive screening method among at-risk populations. Esophageal squamous cell carcinoma (ESCC) and residents of the Asian cancer belt are examples of those malignancies and populations. Previous epidemiological studies using cfDNA have pointed to the need for high volumes of good quality plasma (i.e., $>1 \mathrm{~mL}$ plasma with 0 or 1 cycles of freeze-thaw) rather than archival serum, which is often the main available source of cfDNA in retrospective studies. Here, we have investigated the concordance of TP53 mutations in tumor tissue and cfDNA extracted from archival serum left-over from 42 cases and 39 matched controls (age, gender, residence) in a high-risk area of Northern Iran (Golestan). Deep sequencing of TP53 coding regions was complemented with a specialized variant caller (Needlestack). Overall, $23 \%$ to $31 \%$ of mutations were concordantly detected in tumor and serum cfDNA (based on two false discovery rate thresholds). Concordance was positively correlated with high cfDNA concentration, smoking history $(p$-value $=0.02)$ and mutations with a high potential of neoantigen formation (OR; 95\%CI = $1.9(1.11-3.29))$, suggesting that tumor DNA release in the bloodstream might reflect the effects of immune and inflammatory context on tumor cell turnover. We identified TP53 mutations in five controls, one of whom was subsequently diagnosed with ESCC. Overall, the results showed that cfDNA mutations can be reliably identified by deep sequencing of archival serum, with a rate of success comparable to plasma. Nonetheless, 70\% non-identifiable mutations among cancer patients and $12 \%$ mutation detection in controls are the main challenges in applying cfDNA to detect tumor-related variants when blindly targeting whole coding regions of the TP53 gene in ESCC.
\end{abstract}

Keywords: circulating cell-free DNA; liquid biopsy; circulating tumor DNA; esophageal squamous cell carcinoma; TP53; tumor mutation; variant caller; deep sequencing; neoantigen

\section{Introduction}

Circulating tumor DNA is but a tiny fraction of cfDNA in blood circulation. CfDNA can be extracted from plasma or serum, although plasma is widely recommended as the 
preferable standard media for studying cfDNA [1,2]. However, widespread use of serum for medical workups in clinical and hospital biobanks have made it more easily available than plasma in retrospective case-control studies, and it is the sole available resource in many population-based studies. It is therefore important to investigate whether cfDNA may be successfully retrieved and sequenced from archival collections.

Esophageal squamous cell carcinoma (ESCC), with nearly 440,000 deaths annually [3], has a dismal prognosis [4]. Symptomatic diagnosis is usually late.Due to space availability in the mediastinum, lack of serosa, and flexible tubal structure, tumor mass in the esophagus can expand silently until obstructive symptoms appear. As a result, 5-year survival rates are low (5-15\%) but significantly improve when diagnosis is made at early stages [5]. Notably, a 95\% 5-year survival rate has been reported given early diagnosis [6]. Despite several ongoing efforts for early detection of ESCC in endemic areas [7], a minimally invasive and feasible approach has yet to be developed. Liquid biopsy and its cfDNA component have shown encouraging features in studying treatment response among ESCC patients [8].

TP53 is the most frequently mutated gene in ESCC, with about 90\% of ESCC tumors carrying one or more TP53 mutations in the Asian esophageal cancer belt $[9,10]$. TP53 mutations are detectable in the dysplastic squamous mucosa, highlighting its potential as an early biomarker for esophageal squamous dysplasia and ESCC [11]. Studies have evaluated and reviewed the diagnostic and prognostic value of mutation detection in liquid biopsies of ESCC using next-generation sequencing [12-14]. Though the concordance fraction of serum cfDNA for tumor mutations has not been investigated as thoroughly [15], to our knowledge, published studies have not reported the diagnostic value of serum cfDNA in ESCC.

Over the past few decades, several large case-control studies on ESCC epidemiology in endemic areas have collected serum samples. These archived biosamples may not meet pre-analytical standards for cfDNA evaluation. However, they represent a unique resource associated with large-scale, well-documented studies, which are the cornerstone of our current knowledge on ESCC epidemiology in endemic areas. Therefore, it is of particular interest to investigate their diagnostic value in cfDNA studies.

Here we have applied exon-targeted deep sequencing, coupled with a stringent bioinformatics approach, to detect TP53 mutations in cfDNA from left-over archival sera collected in a case-control study conducted between 2002-2008 in Golestan (Northern Iran). Serum cfDNA TP53 variants were analyzed in 42 ESCC and compared to matched tumor DNA variants previously identified by Sanger sequencing, providing a concordance ratio between cfDNA and tumor DNA. We also assessed cfDNA TP53 variants in 39 matched cancer-free patients.

\section{Results}

A total of 42 ESCC cases and 39 matched controls were initially included in this study. In 22 cases, tumor location was in the mid-third of the esophagus. The bulk of tumor was in the lower third and upper third of the esophagus in 18 and 2 cases, respectively. The mean age of cases and controls were 62 and 64 years old. Half of the controls and $52 \%$ of cases were females. The proportion of smokers among cases $(23.8 \%)$ and controls $(20.5 \%)$ was comparable. The mean of left-over serum volume for cfDNA extraction was $581 \mu \mathrm{L}$ among cases and $880 \mu \mathrm{L}$ among controls ( $p$-value $=0.00001$ ). After excluding 3 outliers for cfDNA level $(>100 \mathrm{ng} / \mathrm{mL}$ ) the mean concentration of cfDNA among neighborhood controls and cases were 1.62 and $1.18 \mathrm{ng} / \mathrm{mL}$ ( $p$-value $=0.06$ ), respectively. Targeted deep sequencing readouts were analyzed using Needlestack, a multi-sample variant caller designed for the detection of low abundance mutations. Mean sequencing coverages for first and second technical duplicates were 557,735 and 596,654 reads among cases, respectively, and 572,085 and 532,498 reads among controls, respectively. Total reads in duplicates were less than 100 for one ESCC case and three controls. Figure 1 depicts the distribution of cfDNA fragments in an ESCC case after applying size-selection using magnetic beads. 

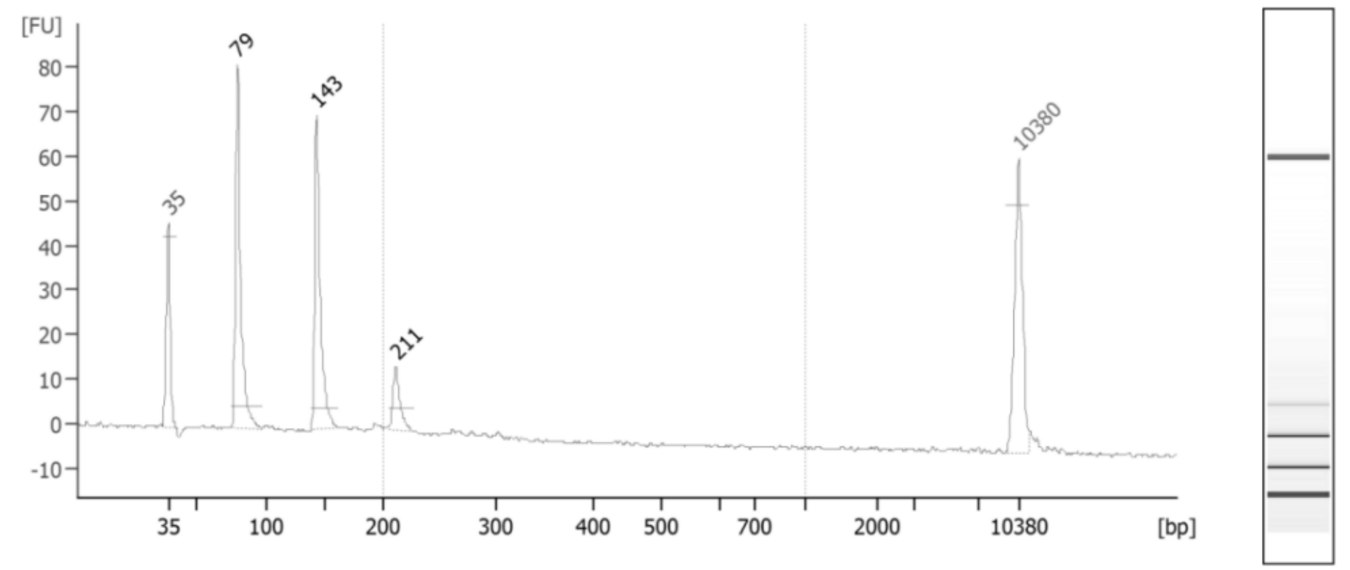

Figure 1. CfDNA fragments extracted from the serum of a randomly-selected ESCC case. The average size is $220 \mathrm{bp}$, showing several fragments under $200 \mathrm{bp}$ (start and end picks are the standard markers). Long fragments (between 200 and $1000 \mathrm{bp}$ ) consisted of 11\% of total serum cfDNA.

We observed a concordance between TP53 variants previously detected in FFPE tumor biopsy and those detected by deep-sequencing in serum cfDNA in 10 cases ( $24 \%$ concordance), among which, in five cases, TP53 variants were observed in both technical duplicates. By decreasing the Q-value threshold from 50 to 20, cfDNA variants in additional five ESCC cases were in concordance with tumor mutations (36\%). Among them, in eight cases, TP53 variants were observed in both technical duplicates. Figure 2 depicts the effect of varying Q-value thresholds on detecting total TP53 variants in cfDNA among cases and controls, regardless of TP53 alteration in tumor tissue.

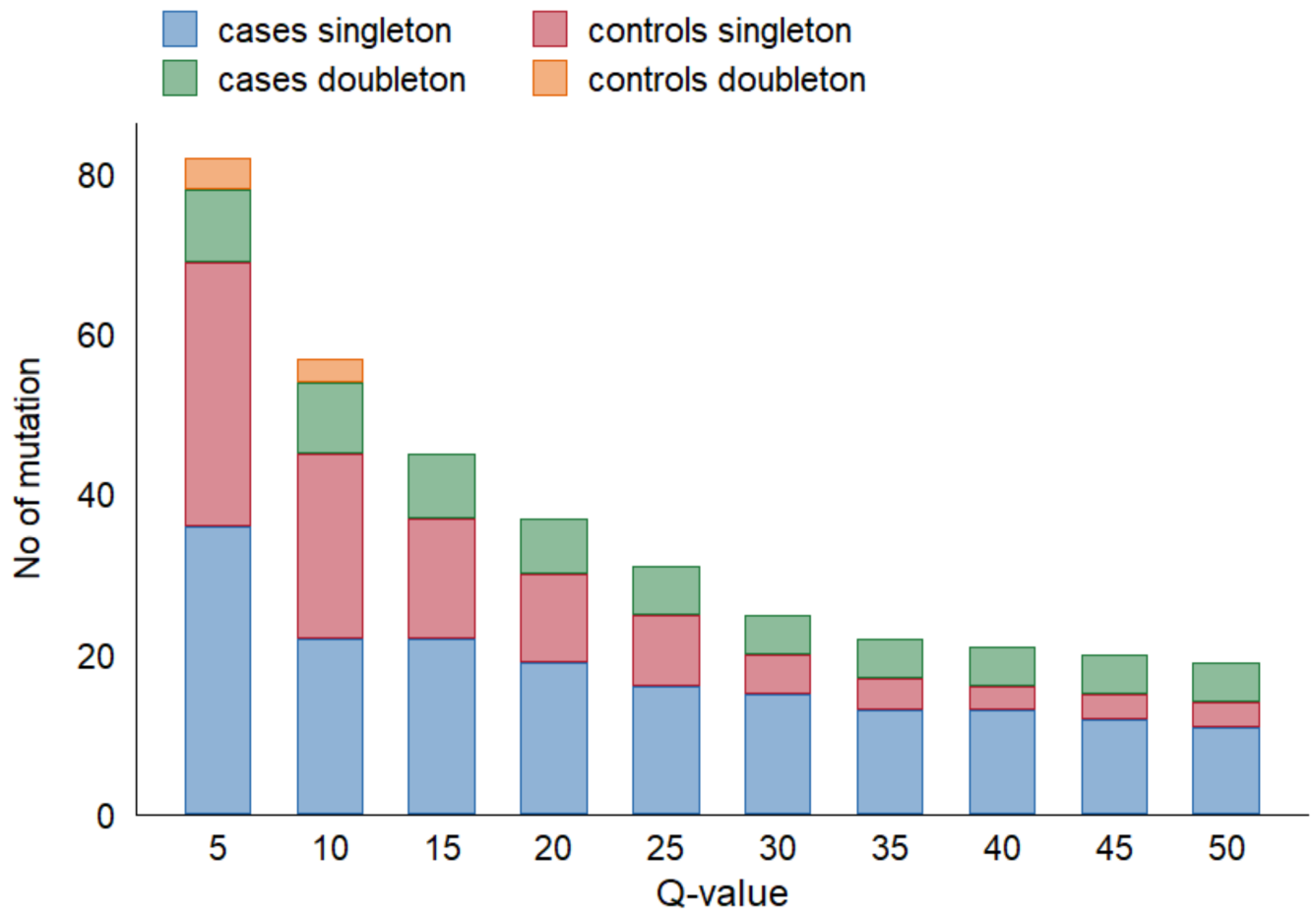

Figure 2. Effect of Q-value threshold on detecting TP53 variants in cfDNA.

We searched for TP53 variants in cfDNA, matching the pool of positions and base changes detected in TP53 across all ESCC tumors included in our study. Among the 42 cases, cfDNA variants matched with tumor variants in 13 patients (31\% concordant, 8 duplicate). Among the 39 controls, we observed five single TP53 variants in six age-sex-matched 
neighborhood controls. None of these TP53 variants were detected in both technical duplicates. During follow-up, one of six controls with $7577079 \mathrm{C}>\mathrm{T}$ variant was diagnosed with ESCC less than a year after enrollment. Another control, harboring the same mutation in cfDNA, was alive without any sign of malignancy 15 years after enrollment. The cause of mortality amongst remaining controls with TP53 variants in cfDNA (7577121G $>$ T, $7577509 \mathrm{C}>\mathrm{T}, 7577536 \mathrm{~T}>\mathrm{A}, 7577580 \mathrm{~T}>\mathrm{C}$ ) was not cancer-related and the death date was between 6 and 10 years after enrollment.

Table 1 compares primary exposure data, total DNA, and sequencing performance among cases and controls. Drinking and smoking are two major risk factors for ESCC. In the study area, drinking habits are uncommon, and opium use is an established risk factor for ESCC. A total of $63 \%$ of ESCC cases with detected concordant cfDNA and $29 \%$ of cases without detectable concordant cfDNA were either tobacco or opium users $(p$-value $=0.04$ ).

Table 1. Characteristics of cases and controls with and without concordant TP53 cfDNA variants, comparing TP53 mutations in FFPE tumor tissues.

\begin{tabular}{|c|c|c|c|c|c|c|}
\hline \multirow[t]{2}{*}{ Variables } & \multicolumn{2}{|c|}{ ESCC Cases } & \multicolumn{4}{|c|}{ Healthy Controls } \\
\hline & cfDNA+ & cfDNA- & $p$-Value & cfDNA+ & cfDNA- & $p$-Value \\
\hline Number & $14^{1}$ & 29 & & $5^{1}$ & 33 & \\
\hline Mean age(SD) & $62(9)$ & $63(11)$ & 0.72 & $69(16)$ & $63(9)$ & 0.23 \\
\hline Sex (Female \%) & $44 \%$ & $45 \%$ & 0.75 & $57 \%$ & $59 \%$ & 0.25 \\
\hline Ever-smoker $(\%)$ & $45 \%$ & $16 \%$ & 0.02 & $22 \%$ & $18 \%$ & 0.52 \\
\hline Chewing tobacco (\%) & $27 \%$ & $10 \%$ & 0.07 & $0 \%$ & $3 \%$ & 0.06 \\
\hline Ever-opium user (\%) & $35 \%$ & $26 \%$ & 0.49 & $28 \%$ & $15 \%$ & 0.41 \\
\hline Median cfDNA (ug) & 179 & 72 & 0.02 & 165 & 139 & 0.72 \\
\hline Mean sequencing coverage & 670,174 & 550,847 & 0.02 & 668,927 & 533,174 & 0.10 \\
\hline
\end{tabular}

${ }^{1}$ Healthy control who developed ESCC after the enrollment regrouped as a new case.

Figure 3 depicts the positions of TP53 variants in cfDNA among healthy controls and ESCC cases. The genomic positions of TP53 mutations in our series of ESCC tumors were mostly in exons 5-8. TP53 variants in serum cfDNA showed a similar pattern. Among five cfDNA TP53 variants identified in controls, 7577509C >T (E258K) was considered pathogenic (CLINSIG database) with a REVEL score of 0.96 . The mean allelic fraction of variants in controls' cfDNA was $0.19 \%$ and ranged from $0.01 \%$ to $0.42 \%$. After excluding one ESCC case with a higher than $10 \%$ allelic fraction, cfDNA allelic fraction among cases with concordant TP53 mutations ranged from $0.04 \%$ to $0.94 \%$, with a mean of $0.27 \%$. The allelic fraction of concordant TP53 mutations in serum cfDNA was not different between cases and controls ( $p$-value $=0.52$ ).

We examined the nucleotide distance between genetic positions of mutations across TP53 gene in ESCC tumor tissues and categorized them into three groups; less than 5 nucleotides distance between adjacent variants ( 15 variants), between 5 and 10 nucleotides (7 variants), and more than ten nucleotides distance between variants (22 variants). The mean detection fraction of cfDNA concordant variants decreased from $50 \%$ to $20 \%$ as genetic coordinates between mutations decreased (Figure 4). The mean of cfDNA allelic fractions was similar among groups (mean allelic fraction $=0.2$ ). 


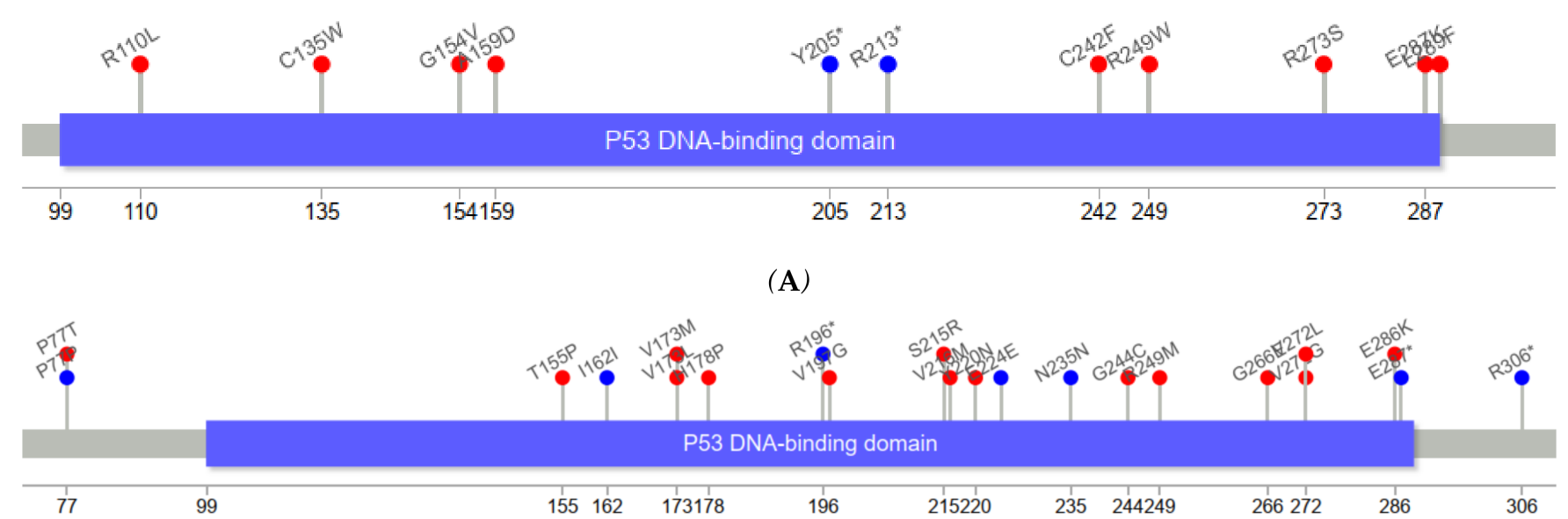

(B)

Figure 3. Visualization of mutation (mutations that have no record for protein data not included) [16] (A) TP53 variants detected in cfDNA of ESCC cases concordant with a mutation in tumor tissue (R110L, C135W, G154V, A159D, Y205*, R213*, C242F, R249W, R273S, E287K, L289F) (B) TP53 variants in tumor tissues not detected in cfDNAs of ESCC cases (P77T, P77P, T155P, I162I, V173M, V173L, H178P, R196*, V197G, S215R, V216M, Y220N, E224E, N235N, G244C, R249M, G266E, V272L, V272G, E286K, E287*, R306*) Supplementary Tables S1 and S2 contain the observed mutations in cfDNA and tumor tissues. Nonsynonymous mutations (red circle), synonymous mutation (blue circle).
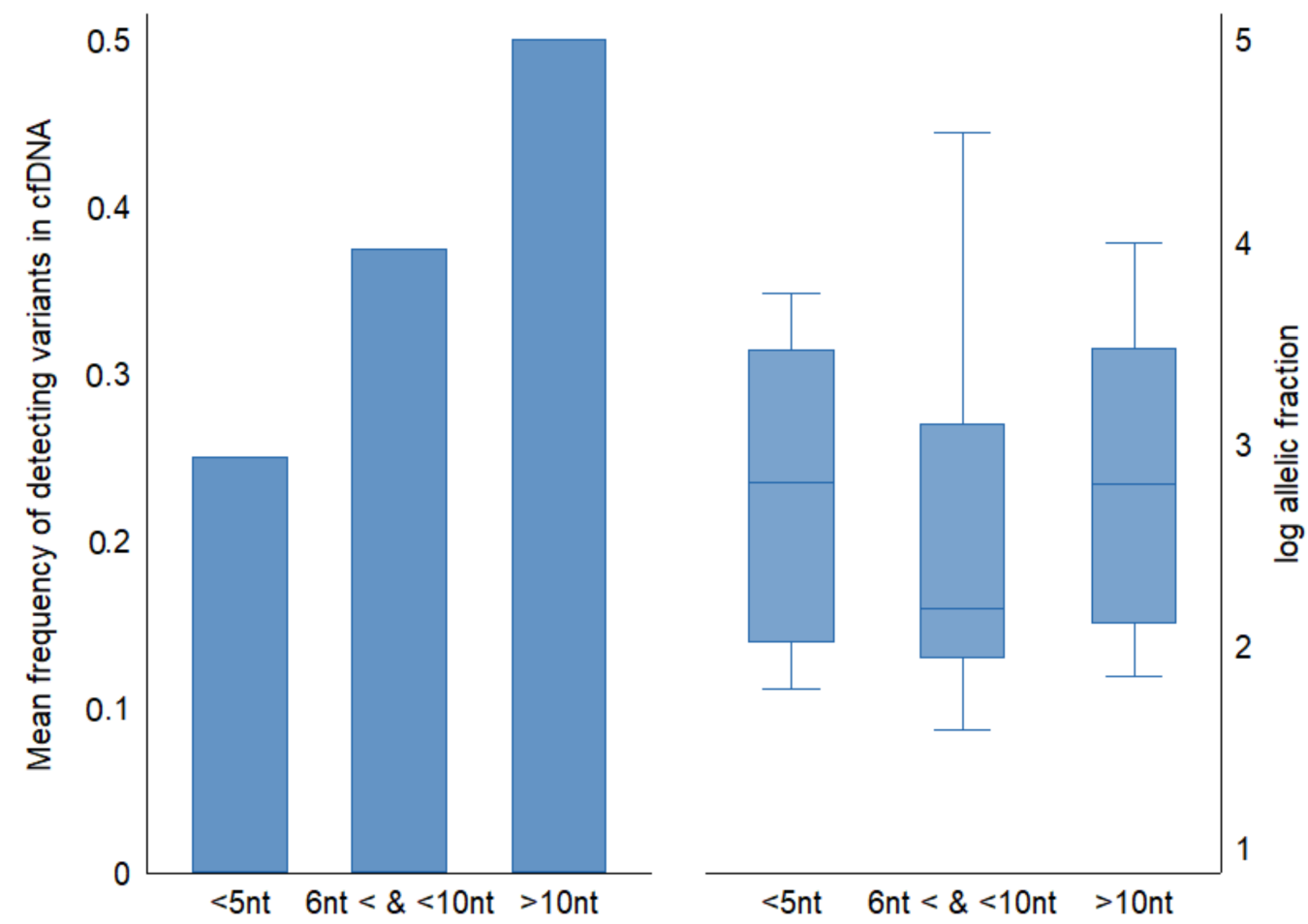

Figure 4. Comparison of frequency of detecting concordant variants in cfDNA relative to nucleotide distance between genetic coordinates of mutations in FFPE tumor tissue. Half of ESCC tumor TP53 mutations consisted of adjacent variants ( $<10 \mathrm{nt}$ apart from the next mutation). Allelic fractions across groups were similar.

We evaluated the frequency of tumor-specific neoantigen formation using predictions from the TCGA database [17]. In the logistic regression model with the detectability of tumor TP53 mutations in cfDNA as an outcome, we observed higher detection rate of mutations with higher frequency of neoantigen formation (Supplementary Table S3). The 
associated probability of TP53 mutation detection in cfDNA did not change when adjusting for the number of HLA subtypes (Table 2, Supplementary Figure S1).

Table 3 summarizes the characteristics of detected and undetected mutations in cfDNA. We did not observe a statistical difference between variant characteristics in relation to their detectability of tumor mutation in cfDNA.

We completed a follow-up study for 26 controls (out of 39) and 36 ESCC cases (out of 42). Staging data was not available for most cases (66\%). For those with available clinical staging data, stage III was the most common. Variant detection in cfDNA did not associate with different survival rates (hazard ratio for detection of concordant cfDNA variants (95\%CI): $1.17(0.49-2.80))$. In 15 years of follow-up, four healthy controls developed cancers (two ESCC, one lymphoma and one skin cancer). One control with pathogenic TP53 variant in cfDNA was diagnosed with ESCC six months after recruitment. Self-report of chronic diseases and inflammatory conditions (arthritis, cardiovascular disease, history of stroke, diabetes, COPD, renal failure, and hepatitis) were absent among controls with detectable TP53 mutations in the serum cfDNA. In contrast, six controls (19\%) with no TP53 cfDNA variants reported one or more of the above-mentioned conditions.

Table 2. Odds of detecting TP53 mutations in cfDNA relevant to the frequency of neoantigen formations resulted from mutations.

\begin{tabular}{ccccc}
\hline $\begin{array}{c}\text { Neoantigen Formation } \\
\text { Frequency }\end{array}$ & $\begin{array}{c}\text { Protein Variants } \\
\text { from Detected } \\
\text { Mutations in cfDNA }\end{array}$ & $\begin{array}{c}\text { Protein Variants } \\
\text { from Undetected } \\
\text { Mutations in cfDNA }\end{array}$ & $\begin{array}{c}\text { Unadjusted OR } \\
\text { (95\%CI) }\end{array}$ & $\begin{array}{c}\text { Adjusted OR for HLA } \\
\text { Frequency (95\%CI) }\end{array}$ \\
\hline 1st quantile & $71(49 \%)$ & $74(51 \%)$ & Reference & Reference \\
2nd quantile & $130(55 \%)$ & $106(45 \%)$ & $1.28(0.84-1.93)$ & $1.29(0.85-1.95)$ \\
3rd quantile & $123(53 \%)$ & $109(47 \%)$ & $1.17(0.77-1.78)$ & $1.22(0.80-1.85)$ \\
4th quantile & $131(61 \%)$ & $84(39.1 \%)$ & $1.62(1.06-2.48)$ & $1.91(1.11-3.29)$ \\
\hline
\end{tabular}

Table 3. Characteristics of tumor TP53 variants according to detectability of variants in cfDNA cases and controls.

\begin{tabular}{|c|c|c|c|}
\hline \multirow[b]{2}{*}{ Variant Characteristics } & \multicolumn{2}{|c|}{ ESCC Tumor TP53 Variants } & \multirow[b]{2}{*}{$\begin{array}{l}\text { Detected in } \\
\text { Control cfDNA }\end{array}$} \\
\hline & $\begin{array}{l}\text { Detected in } \\
\text { Case cfDNA }\end{array}$ & $\begin{array}{l}\text { Undetected in } \\
\text { Case cfDNA }\end{array}$ & \\
\hline Unique variants & 14 & 29 & 5 \\
\hline Duplicate variants & 1 & 5 & 0 \\
\hline \multicolumn{4}{|l|}{ Variant categories } \\
\hline intronic & $1(7 \%)$ & 0 & 0 \\
\hline missense & $10(72 \%)$ & $25(72 \%)$ & $5(100 \%)$ \\
\hline nonsense & $3(21 \%)$ & $4(11 \%)$ & 0 \\
\hline silent & 0 & $3(8.5 \%)$ & 0 \\
\hline splice & 0 & $3(8.5 \%)$ & 0 \\
\hline Variants in hotspots & $12(86 \%)$ & $21(70 \%)$ & $4(80 \%)$ \\
\hline Mean REVEL score (SD) & $0.87(0.1)$ & $0.84(0.2)$ & $0.87(0.2)$ \\
\hline Variants at cpg cites & $4(29 \%)$ & $5(14 \%)$ & $1(20 \%)$ \\
\hline Variants at nucleosome main peak positions [18] & $10(45 \%)$ & $19(58 \%)$ & 0 \\
\hline \multicolumn{4}{|l|}{ Mutation type } \\
\hline $\mathrm{A}: \mathrm{T}>\mathrm{C}: \mathrm{G}$ & $2(14 \%)$ & $6(17 \%)$ & $1(20 \%)$ \\
\hline $\mathrm{A}: \mathrm{T}>\mathrm{G}: \mathrm{C}$ & 0 & $1(3 \%)$ & 0 \\
\hline $\mathrm{A}: \mathrm{T}>\mathrm{T}: \mathrm{A}$ & $1(7 \%)$ & $1(3 \%)$ & 0 \\
\hline $\mathrm{G}: \mathrm{C}>\mathrm{A}: \mathrm{T}$ & $6(43 \%)$ & $18(51 \%)$ & $3(60 \%)$ \\
\hline $\mathrm{G}: \mathrm{C}>\mathrm{C}: \mathrm{G}$ & $1(7 \%)$ & 0 & 0 \\
\hline $\mathrm{G}: \mathrm{C}>\mathrm{T}: \mathrm{A}$ & $4(29 \%)$ & $9(26 \%)$ & $1(20 \%)$ \\
\hline
\end{tabular}

\section{Discussion}

Using targeted deep sequencing of TP53 coding regions (exons 2 to 11 and flanking splicing sites), we demonstrated a $24 \%$ to $36 \%$ concordance fraction between variants detected in cfDNA from archived serum and paired FFPE ESCC tumor tissue. In this 
study, variants were cataloged as a probable true positive if they met either one of the following criteria: concordant detection in technical duplicates or/and concordance with the variant previously detected by Sanger sequencing in FFPE tumor tissue of the same patient. Overall, the concordance rate reported here using archived serum was comparable to the one reported by others using plasma [19], suggesting that serum may represent a valuable source of cfDNA for detecting tumor-associated gene variants, provided that sufficiently sensitive and specific detection methods are used.

Standard preanalytical conditions for detecting mutations in cfDNA recommend the usage of plasma rather than serum, with a volume of or exceeding $1 \mathrm{~mL}$, with a maximum single cycle of freeze-thaw, and storage at $-80{ }^{\circ} \mathrm{C}$ [1]. Our study samples were sera with less than $0.8 \mathrm{~mL}$ available volume (mean $0.5 \mathrm{~mL}$ ), kept between $10-13$ years at $-80{ }^{\circ} \mathrm{C}$ in Golestan Biobank and for two years at $-20{ }^{\circ} \mathrm{C}$ in other research centers, with more than or equal to three cycles of freeze-thaw. Due to the matched study design, preanalytical conditions for cases and controls were similar. The only significant difference was the longer time interval between withdrawing blood and performing centrifuge for neighborhood controls compared to cases in research clinics. The longer time-interval was due to shipment of blood in coolers from distant villages to research center. On-road shipment and longer times prior to serum extraction can cause more lysis and, as a result, we observed marginally but statistically non-significantly higher levels of cfDNA among neighborhood controls compared to ESCC cases. A possible drawback of using serum is the presence of DNA fragments from lysed white blood cells, as a result, serum contains 2-24 times more cfDNA than plasma [20-22], resulting in a smaller proportion of tumorderived DNA fragments in serum cfDNA compared to plasma. To minimize this effect, we included a size selection step before sequencing to reduce the number of long DNA fragments, mostly associated with lysed cells.

Two deep-sequencing approaches have been commonly used to screen for TP53 mutations in cfDNA. Most studies have focused on a limited number of a priori known point mutations, which allow sequencing of a selected library of certain genomic concordances. Other studies (such as ours) have examined entire coding regions, which allow screening for all possible mutations in the range of DNA targeted. The latter method has the advantage of not requiring prior knowledge of the mutational profile. However, its disadvantage over the former is the possibility of differential coverage of the gene regions, thus potentially missing variants located in the region with lower sequencing coverage. A study in head and neck SCC reported a different concordance fraction of plasma cfDNA when using targeted TP53 mutation (32\%) or TP53 coding regions (2.7\%) [19]. In our study, based on a Q-value threshold of 30 a concordance fraction of $24 \%$ was found which is comparable to concordance reported for ESCC in a multigene multi-cancer study [23]. By lowering the Q-value from 50 to 20, the concordance fraction improved to $36 \%$.

Given that TP53 mutations in our selected cases were located over 4 exons in tumor tissue, our approach of sequencing all coding exons may have decreased the probability of detecting mutations due to unnecessary reduction of sequencing coverage. We observed that more than half of the readings were from the exons without corresponding mutations in the tumor. If we ignored our prior knowledge of mutations in tumors and only approved duplicate variants in cfDNA, the concordance between tumors and cfDNA would be $12 \%$ to $19 \%$ (based on the Q-value threshold selected). None of the TP53 variants among controls were duplicates. Based on a handful of studies and a modest number of cases, concordant mutation detection in cfDNA of esophageal cancer cases varies [24], e.g., 14\% using 12 gene panels [17].

Serum cfDNA in $74 \%$ of our ESCC cases did not show detectable TP53 variants, suggesting that detection may be associated with specific factors that are unevenly distributed among cases. Independent from the amount of extracted cfDNA, smoking showed a significant positive association with TP53 detection in cfDNA $(p=0.02)$. Of note, in our study population, tumor TP53 mutations did not show an association with smoking or chewing tobacco [9]. Thus, the higher rate of concordant TP53 variants in cfDNA of tobacco 
smoker/chewer cases suggests that tobacco consumption may be correlated with a higher rate of shedding tumor DNA in the serum. Systemic inflammation induced by smoking may cause increased tumor cell damage and turnover, resulting in the release of tumor DNA in the serum [25]. Alternatively, studies have reported a higher rate of overall clonal hematopoiesis $(\mathrm{CH})$ in smoking-related diseases. In $\mathrm{CH}$, a fraction of white blood cells may carry somatic mutations [26]. Thus, $\mathrm{CH}$ and DNA release from lysed white blood cells might account for the origin of some of the observed variants in cfDNA—which are coincidentally the same as those found in the tumor. However, it remains to be determined if $\mathrm{CH}$ is due to smoking or the general inflammatory process [27].

A total of $45 \%$ of tumor TP53 mutations in our series were recorded in the TCGA-based neoantigen database [17]. Despite the small sample size, we observed a greater probability of detecting tumor TP53 mutations in cfDNA among variants with the highest quartile of neoantigen formation. This observation suggests that tumor cells expressing potentially neoantigenic TP53 variants may be more prone to release tumor DNA in the bloodstream than tumor cells expressing non-antigenic variants. The presence of a neoantigenic variant may specify a different immune microenvironment in the tumor with, perhaps, increased tumor cell turnover and, consequently, tumor DNA release in the bloodstream. As for the association with tobacco usage discussed above, this hypothesis needs further investigation with a larger sample size.

We observed that adjacent variants (less than $5 \mathrm{nt}$ up or downstream) in different samples had a lower chance of being detected in serum cfDNA regardless of their allelic fraction, which explains $26 \%$ of missed TP53 mutations. It could be a random error and we did not determine a reason for it. Among the filtering steps, we removed variants with an allelic fraction of 10 times higher than candidate variants in 5-nt to 10-nt distance from the target candidate [28]. It could indicate that using whole coding region for genes and cancers with prevalent adjacent mutations $(<10 \mathrm{nt})$, may cause some limitations, particularly among variant with too low allelic fraction.

Some studies reported detecting up to $11 \%$ of TP53 variants in cfDNA of non-cancerous controls [29]. In the current study, we did not detect TP53 variants in both technical duplicates among controls, either through matching for known tumor mutations or blind screening of TP53 coding regions. We detected non-duplicate TP53 variants in 6 controls, of which one developed ESCC six months after enrollment. We were unable to verify if the same cfDNA mutation existed in the subsequent esophageal tumor. Given the short time lapse between serum sampling and diagnosis, it is possible that this subject already carried an asymptomatic tumor at the time of recruitment. Alternatively, TP53 mutations can be detected in a small subset of normal esophageal epithelia, as the result of ongoing exposure to environmental risk factors [30]. Entertaining the possibility of field cancerization [31] due to shared exposure to carcinogens might be a plausible explanation, given that neighborhood controls in this study shared a comparable environment with cases [32].

Likewise, we did not observe TP53 mutations in cfDNA of controls who subsequently developed other cancers than ESCC. Of note, one of our study's limitations is that we did not sequence the WBC of these controls to assess the possibility of $\mathrm{CH}$ as the origin of the mutation.

Due to the scarcity of DNA of tumor origin in the bloodstream [33], it is a valid argument to address whether the observed concordant mutations were true variants or false-positive findings resulting from our targeted search for mutations [34]. All patients in our series had a tumor diameter of $>1 \mathrm{~cm}$. It is estimated that, with such tumor size, tumor DNA would represent between $0.1 \%$ and $0.01 \%$ of cfDNA. Notably, all detected TP53 mutations in our studywere identical to those previously found in tumors, with no new TP53 variant. This observation supports that the variants identified in cfDNA in this study are likelyoriginating from the tumor.

This study had several limitations: a small number of ESCC cases, lack of WBC sequencing, use of different sequencing approaches for the tissue and liquid biopsies, and no validation of NGS-detected cfDNA variants with digital droplet PCR or other methods. 
Strengths of the study included: age, sex, residence-matched controls in a populationbased case-control design, follow-up data of controls on cancer occurrence, application of a sensitive method for variant detection, and use of several filtering steps to decrease the probability of false-positive detection.

In conclusion, based on concordance with tumor mutations, archival serum samples appear useful for detecting targeted ESCC tumor TP53 mutations in cfDNA. The implications of our findings are important, echoing the message for mutation-based cancer biomarkers when whole coding regions are blindly screened. This limitation is beyond selection of the type of biological samples (serum or plasma). At the same time, we have shown the importance of ultra-sensitive rare variant callers in avoiding recruiting false positive results. We have also portrayed the biological challenges, e.g., adjacent variants, neoantigens, and certain carcinogenic profiles influencing the specificity of mutation detection in cfDNA. This study, as the first attempt at screening whole coding regions of TP53 amongst one of the highest incident areas for ESCC, reached similar results to those studies which applied the same method in different organs and non-endemic populations. Our study results suggest that, in certain subgroups of at-risk populations (e.g., tobacco users), and in the presence of neoantigens, the probability of detecting TP53 variants in circulation will increase. This serves as a practical application of this study. With due caution, in light of the small study numbers, TP53 mutations in serum cfDNA from tobacco users and mutations with a higher frequency of neoantigen formation were more likely to be detected.

\section{Materials and Methods}

\subsection{Ethical Approval}

This study was approved by the ethical committee of the Digestive Disease Research Institute of Tehran University of Medical Sciences, Tehran, Iran (IRB00001641, 11 January 2003), and Institutional Review Board of National Cancer Institute, Bethesda, MD, USA (NCT00339742, 25 March 2003), and IARC Ethics Committee (project No. 17-30).

\subsection{Study Population, Sample Selection}

The main study's details were reported earlier [9,35]. Briefly, case subjects were recruited at Atrak clinic, the only specialized clinic for esophageal cancer diagnosis in eastern Golestan, from 2003 to 2008. Included cases were histopathologically confirmed ESCC patients who underwent upper gastrointestinal endoscopy and agreed to participate in the study. Biopsy specimens were oriented, fixed in $10 \%$ buffered formalin, embedded in paraffin, sectioned, stained with hematoxylin and eosin, and examined by experienced pathologists (M. Sotoudeh and B. Abedi-Ardekani). Two population-based control subjects were selected, individually matched to the cases by age ( \pm 2 years), sex, and location (i.e., from the same neighborhood or village). In total, we recruited 300 cases and 571 matched controls. After obtaining written informed consent, a nurse and a physician administered a structured questionnaire. No proxies were used. Data were collected on demographic variables, lifelong history of tobacco, opium, alcohol use, medication, and several potential confounders. Before endoscopy, a $12 \mathrm{~mL}$ venous blood sample was collected from each case subject. The serum was immediately separated into $5 \mathrm{~mL}$ EDTA-contained tubes, aliquoted, and stored at $-80^{\circ} \mathrm{C}$. Collected blood samples from matched controls were transferred on ice in a cooler box $\left(\sim 4{ }^{\circ} \mathrm{C}\right)$. The time between collection and processing of neighborhood control samples was $<12 \mathrm{~h}$. This time interval was significantly higher for controls than ESCC cases, which was unavoidable due to the lack of laboratory facilities in the more than 200 villages visited while collecting neighborhood controls. We followed-up on cases and controls for the current study through a linkage to local cancer registry data updated until 2020. We also tried contacting neighborhood controls by phone to investigate their medical status.

Details of the analysis of TP53 mutations in ESCC patients have been reported elsewhere by Abedi-Ardekani et al. [9]. In brief, DNA from formalin-fixed paraffin-embedded 
biopsy tissues with tumor purity of $>70 \%$ was extracted using QIAamp DNA MicroKit from Qiagen (Hilden, Germany). For a total of 119 ESCC cases, the TP53 coding region (exons 2-11) went through sequencing using Applied Biosystems PRISM 3100 Genetic Analyzer (Applied Biosystems, Foster City, CA, USA) in 2011. In total, 120 TP53 mutations were detected in 107 ESCC cases. Of which 101 mutations were in exons 5-8.

Out of 107 ESCC cases with available data on tumor TP53 mutations, we selected 30 ESCC cases based on an in silico experiment. We used a database of TP53 variants in cfDNA of a series of patients with small-cell lung cancer to determine the error rate and proportion of false-positive detection per each genomic concordance [28]. We have added 12 further cases with more than one TP53 mutations in tumors to our selected cases. We included 39 matched controls with available serum samples in our assay.

\subsection{CfDNA Sequencing}

CfDNA was extracted from less than $1 \mathrm{~mL}$ serum using QIAamp DNA circulating Nucleic acid kit (Qiagen, Hilden, Germany). Twenty-seven amplicons with 81 to $139 \mathrm{bp}$ sizes were designed (Eurofine Genomics, Ebersberg, Germany) to cover the coding region of TP53 (exons 2-11) and splicing sites. We prepared two plates in duplicate, containing equal molar DNA of $5 \mathrm{ng}$. We vacufuged 24 samples and concentrated DNA to reach the $10 \mathrm{uL}$ reaction volume. Then, plates were dried out through heating at $65^{\circ} \mathrm{C}$ for $2 \mathrm{~h}$. We used GeneRead DNAseq Panel PCR kit V2 (Qiagen, Paris, France) for multiplex PCR to enrich the targets. PCR Mix consisted of $2 \mathrm{uL}$ buffer, $1 \mathrm{uL}$ primer pool $(0.6 \mu \mathrm{M})$, and $6.27 \mathrm{uL}$ water. PCR reaction was $95^{\circ} \mathrm{C}(15 \mathrm{~min})$ and 29 cycles of $95^{\circ} \mathrm{C}(15 \mathrm{~s}), 60^{\circ} \mathrm{C}(2 \mathrm{~min})$, and $72{ }^{\circ} \mathrm{C}$ (10 min). Primers were diluted to $100 \mu \mathrm{M}$ each and pooled in an equimolar way (1.85 $\mu \mathrm{M}$ final concentrations).

We used two quality controls: (1) a positive plasma control with known TP53 mutation in cfDNA (Lung cancer sample RS113032 with Chr17:7576897G>A variant); (2) a pooled serum of 15 ESCC cases were divided into three duplicates (6 samples) without prior knowledge about the mutational profile of cases.

After amplification, we purified $10 \mathrm{uL}$ of PCR product with $18 \mathrm{uL}$ Serapure beads (ThermoFisher Scientific, Strasbourg, France). After purification, eight random samples were quantified using Qubit HS-ds DNA kit (Invitrogen, ThermoFisher Scientific, Strasbourg, France). The mean of DNA was 10 ng/uL. NEBNext Library Prep Set for Ion Torrent (New England BioLabs, Paris, France) with an in-house made P1 adaptor was prepared following manufacturer's instructions. At this stage, individual barcodes were added, and amplicons were end-repaired and ligated to the adaptors. An additional step of bead purification with 1.8 ratios was carried out. A brief step of 4 cycles of amplicon amplification was performed through PCR reaction of $93^{\circ} \mathrm{C}(30 \mathrm{~s}), 98^{\circ} \mathrm{C}(10 \mathrm{~s}), 58^{\circ} \mathrm{C}(30 \mathrm{~s})$, and four cycles of $65^{\circ} \mathrm{C}(30 \mathrm{~s})$ and a final step of $5 \mathrm{~min}$ at $65^{\circ} \mathrm{C}$. Total PCR reaction volume was $25 \mathrm{uL}$, including $11 \mathrm{uL}$ DNA. The second step of DNA quantification was done on another set of 8 random cases, and all samples had ranges between 11-17 ng/uL DNA. A total of $22 \mathrm{uL}$ volume per well was left after purification and end-repair. All samples were pooled in an equimolar (assuming $10 \mathrm{ng} / \mathrm{uL}$ DNA in each well). We mixed $60 \mathrm{uL}$ of pooled libraries with $12 \mathrm{uL}$ of $6 \times$ loading dye and loaded those in 5 lanes of $2 \%$ agarose gel ( $20 \mathrm{uL}$ per lane) for size selection. We ran the gel at $150 \mathrm{v}$ for $90 \mathrm{~min}$. After migration, bands with a size of $200 \mathrm{bp}$ to $300 \mathrm{bp}$ were cut and purified using MiniElute gel extraction kit (Qiagen, Paris, France). To verify the process of size selection and dimers' absence, we ran 10 samples on the Bioanalyzer 2100 platform (Agilent Technologies, Santa Clara, CA, USA). Libraries were enriched using emulsion PCR on Ion Sphere particles followed by magnetic bead purification. Libraries were deep sequenced, targeting 10,000 depth on the Ion Torrent ${ }^{\mathrm{TM}}$ Proton Sequencer using Ion TM Hi-Q ${ }^{\mathrm{TM}}$ Sequencing 200 kit and Ion PI v3 (Thermo Fisher Scientific, Waltham, MA, USA). 


\subsection{Sequencing Data Analysis}

We used Needlestack, a multi-sample variant caller designed for the detection of low abundance mutations. Details of the algorithm were mentioned in its methodological paper [28]. Briefly, needlestack estimated the sequencing error rate, at a particular position for a particular base change, using a negative binomial regression (NB) [36]. Then, it detected the true DNA mutations as being outliers of this model of errors. In this method, for each variant, a $p$-value would be dedicated to measuring the probability that a variant is part of the sequencing error model (regression outlier) (Supplementary Figure S2). Due to the requirement of multiple samples to estimate this error model, the individual $p$-value was finally corrected for multiple testing and presented as Q-value in the Phred scale. In order to boost the precision of our method, and because Needlestack should correct only for sequencing artefacts, we used post-calling filters to remove potential remaining errors. These filters included: strand bias variants using relative variant strand bias (RVSB) $<0.85$, and removing adjacent $10 \times$ lower allelic fraction variants within $5 \mathrm{nt}$ region upstream or downstream of the candidate variants in order to remove artefacts from the alignment step. Figure 5 shows the proportion of losing variants after applying these filtering steps.

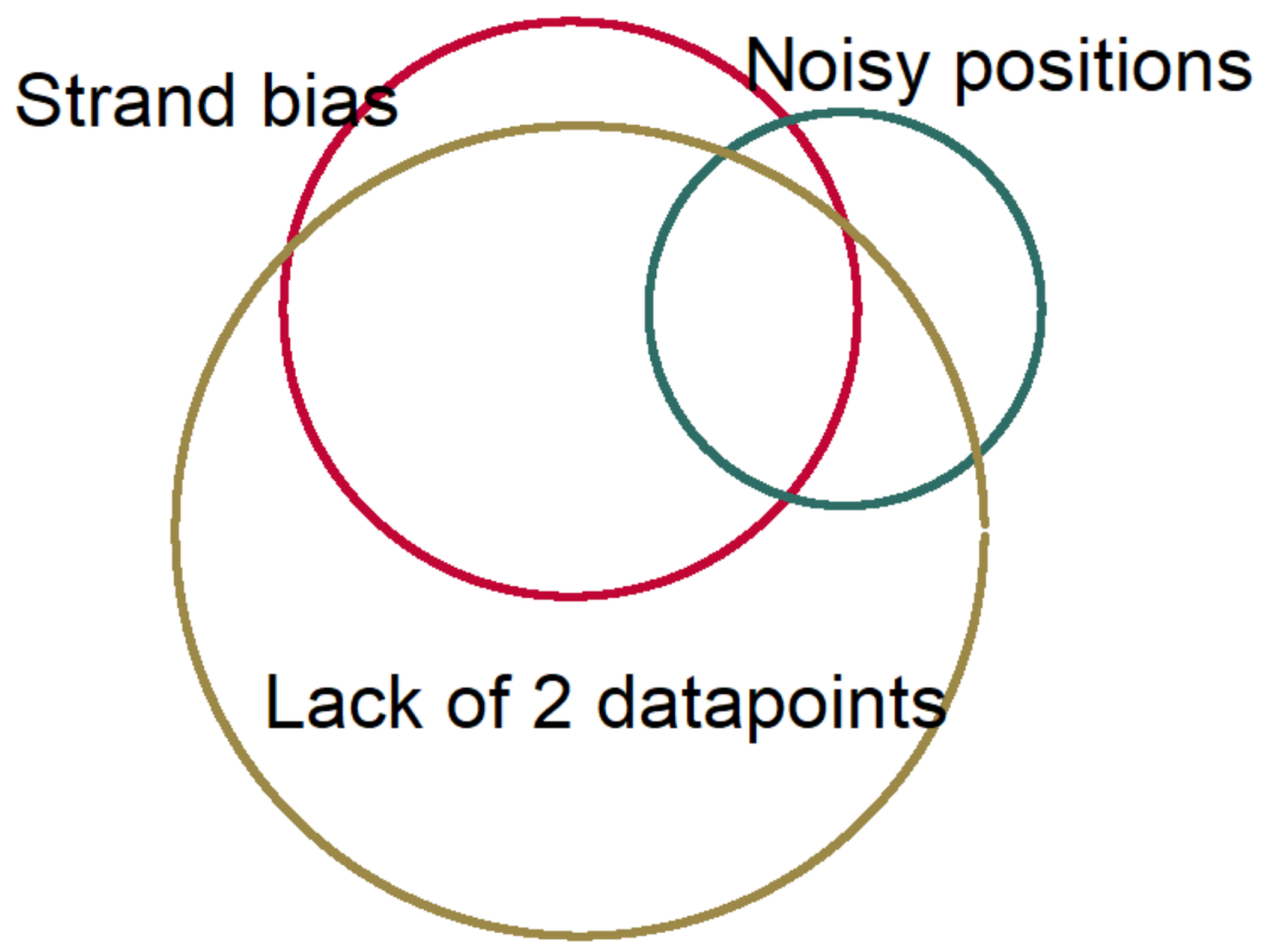

Figure 5. The relative proportion of losing data in each filtering step.

We based our variant detection in cfDNA on the agreement of two or more data points on the presence of mutations to increase the confidence of detecting true positives. Our data points consisted of prior knowledge of mutations in tumors and/or variants' presence in both technical duplicates of the same sample. Two analysis methods were applied: (1) using a priori knowledge of TP53 mutations in tumor FFPE samples and searching for the same mutations in cfDNA variants of cases and controls; (2) ignoring the known mutations in ESCC tumors in our series of cases and searching for duplicate mutations in cases and controls. We used a list of TP53 driver mutations from a large case-series of ESCC tumors in endemic areas (480 ESCC cases) and examined the matched cfDNA variants in cases and controls for the sensitivity analysis. The fraction of observed identical genomic coordinates (position and base change) in the same individual tumor and cfDNA to all tumor mutations was considered a concordance fraction of cfDNA variant detection. Nonparametric statistical tests were done using Stata 14 (StataCrop. LLC, College Station, TX, USA). Cox regression model and log-rank tests were applied for survival analysis. 
Supplementary Materials: The following are available online at https://www.mdpi.com/article/10 .3390 /ijms22115627/s1, Table S1: List of TP53 variants detected in esophageal tumor FFPE tissue which were not detected in serum cfDNA, Table S2: List of TP53 variants were concordantly detected in archival serum cfDNA and esophageal tumor tissue biopsy, Table S3: Frequency of neoantigen formation among TP53 variants detected in several organ malignancies (Bladder, breast, head\&neck, prostate, colorectal, stomach, kidney, liver, and lung) matched with the variants in the Golestan ESCC study, Figure S1: regression graphs of cfDNA mutations, Figure S2: No statistical difference in HLA distribution of neoantigen formation of tumor TP53 variants relevant to detectibelity in cfDNA. Boxplot shows higher frequency of neoantigen formations among cfDNA detected TP53 mutations.

Author Contributions: Conceptualization: B.A.-A., M.F., J.M., R.M., D.N., M.S., H.P., F.S., P.B.; methodology: D.N., T.M.D., B.A.-A., J.M., M.F.; laboratory experiments: D.N., P.H.A.; sequence and statistical data analysis: D.N.; follow-up study: G.R., D.N.; manuscript—writing: D.N.; manuscriptreview and editing: P.H., B.A.-A., R.M., T.M.D., P.H.A. All authors have read and agreed to the published version of the manuscript.

Funding: This project was funded by a grant from the National Institute for Medical Research Development (NIMAD), Iran (Grant\# 977009), and the internal budget of IARC. T.M.D was fully supported by the French National Ligue Against Cancer. D.N. was fully supported by JVA31108425-1, CRUK grand challenge, UK.

Institutional Review Board Statement: The local ethical committee approved the Golestan casecontrol study in the Digestive Disease Research Centre of Tehran University of Medical Sciences, Iran (IRB00001641), Institutional Review Board of National Cancer Institute (NCT00339742, 25 March 2003). IARC Ethics Committee approves the current study under project No. 17-30.

Informed Consent Statement: Informed consent was obtained from all subjects involved in the main case-control study.

Data Availability Statement: Not applicable.

Acknowledgments: We thank Amelie Chabrier for her help in the laboratory arrangement. We thank Atrak Clinic Saman Fahimi, Farhad Islami, Ramin Shakeri, Noushin Taghavi, Haji-Amin Marjani, Rabaeh Rajabzadeh, Monireh Badakhshan, Bita Mohammadi, Halimeh Eskandarnejhad, Safora Kor, Soleiman Kasalkheh, Ashor Yolmeh; and Ahmad Nosrati for their valuable help. We thank the local health networks and health workers (Behvarzes) in the study area for their assistance in recruitment of controls.

Conflicts of Interest: The authors declare no conflict of interest.

Disclaimer: Where authors are identified as personnel of the International Agency for Research on Cancer/World Health Organization, the authors alone are responsible for the views expressed in this article, and they do not necessarily represent the decisions, policy, or views of the International Agency for Research on Cancer/World Health Organization.

\section{References}

1. Merker, J.D.; Oxnard, G.R.; Compton, C.; Diehn, M.; Hurley, P.; Lazar, A.J.; Lindeman, N.; Lockwood, C.M.; Rai, A.J.; Schilsky, R.L.; et al. Circulating Tumor DNA Analysis in Patients With Cancer: American Society of Clinical Oncology and College of American Pathologists Joint Review. J. Clin. Oncol. 2018, 36, 1631-1641. [CrossRef] [PubMed]

2. Pös, Z.; Pös, O.; Styk, J.; Mocova, A.; Strieskova, L.; Budis, J.; Kadasi, L.; Radvanszky, J.; Szemes, T. Technical and Methodological Aspects of Cell-Free Nucleic Acids Analyzes. Int. J. Mol. Sci. 2020, 21, 8634. [CrossRef]

3. Kamangar, F.; Nasrollahzadeh, D.; Safiri, S.; Sepanlou, S.G.; Fitzmaurice, C.; Ikuta, K.S.; Sartorius, B.; Collaborators GOC. The global, regional, and national burden of oesophageal cancer and its attributable risk factors in 195 countries and territories, 1990-2017: A systematic analysis for the Global Burden of Disease Study 2017. Lancet Gastroenterol. Hepatol. 2020, 5, 582-597. [CrossRef]

4. Mir, M.R.; Rajabpour, M.V.; Delarestaghi, M.M.; Hadji, M.; Harirchi, I.; Mir, P.; Mir, A.; Lashkari, M.; Zendehdel, K. Short- and Long-Term Survival of Esophageal Cancer Patients Treated at the Cancer Institute of Iran. Dig. Surg. 2013, 30, 331-336. [CrossRef] [PubMed]

5. Mariette, C.; Markar, S.R.; Dabakuyo-Yonli, T.S.; Meunier, B.; Pezet, D.; Collet, D.; D'Journo, X.B.; Brigand, C.; Perniceni, T.; Carrère, N.; et al. Hybrid Minimally Invasive Esophagectomy for Esophageal Cancer. N. Engl. J. Med. 2019, 380, 152-162. [CrossRef] [PubMed] 
6. Nishizawa, T.; Suzuki, H. Long-Term Outcomes of Endoscopic Submucosal Dissection for Superficial Esophageal Squamous Cell Carcinoma. Cancers 2020, 12, 2849. [CrossRef]

7. Roshandel, G.; Merat, S.; Sotoudeh, M.; Khoshnia, M.; Poustchi, H.; Lao-Sirieix, P.; Malhotra, S.; O’Donovan, M.; Etemadi, A.; Nickmanesh, A.; et al. Pilot study of cytological testing for oesophageal squamous cell dysplasia in a high-risk area in Northern Iran. Br. J. Cancer 2014, 111, 2235-2241. [CrossRef]

8. Azad, T.D.; Chaudhuri, A.A.; Fang, P.; Qiao, Y.; Esfahani, M.S.; Chabon, J.J.; Hamilton, E.G.; Yang, Y.D.; Lovejoy, A.; Newman, A.M.; et al. Circulating Tumor DNA Analysis for Detection of Minimal Residual Disease After Chemoradiotherapy for Localized Esophageal Cancer. Gastroenterology 2020, 158, 494-505.e6. [CrossRef] [PubMed]

9. Abedi-Ardekani, B.; Kamangar, F.; Sotoudeh, M.; Villar, S.; Islami, F.; Aghcheli, K.; Nasrollahzadeh, D.; Taghavi, N.; Dawsey, S.M.; Abnet, C.C.; et al. Extremely High Tp53 Mutation Load in Esophageal Squamous Cell Carcinoma in Golestan Province, Iran. PLoS ONE 2011, 6, e29488. [CrossRef]

10. Zhang, S.; Lu, X.; Hu, C.; Li, Y.; Yang, H.; Yan, H.; Fan, J.; Xu, G.; Abnet, C.C.; Qiao, Y. Serum Metabolomics for Biomarker Screening of Esophageal Squamous Cell Carcinoma and Esophageal Squamous Dysplasia Using Gas Chromatography-Mass Spectrometry. ACS Omega 2020, 5, 26402-26412. [CrossRef]

11. Chen, X.X.; Zhong, Q.; Liu, Y.; Yan, S.M.; Chen, Z.H.; Jin, S.Z.; Xia, T.-L.; Li, R.-Y.; Zhou, A.-J.; Huang, Y.-H.; et al. Genomic comparison of esophageal squamous cell carcinoma and its precursor lesions by multi-region whole-exome sequencing. Nat. Commun. 2017, 8, 524. [CrossRef]

12. Matsushita, D.; Arigami, T.; Okubo, K.; Sasaki, K.; Noda, M.; Kita, Y.; Mori, S.; Uenosono, Y.; Ohtsuka, T.; Natsugoe, S. The Diagnostic and Prognostic Value of a Liquid Biopsy for Esophageal Cancer: A Systematic Review and Meta-Analysis. Cancers 2020, 12, 3070. [CrossRef] [PubMed]

13. Gormally, E.; Caboux, E.; Vineis, P.; Hainaut, P. Circulating free DNA in plasma or serum as biomarker of carcinogenesis: Practical aspects and biological significance. Mutat. Res. 2007, 635, 105-117. [CrossRef] [PubMed]

14. Jahangiri, L.; Hurst, T. Assessing the Concordance of Genomic Alterations between Circulating-Free DNA and Tumour Tissue in Cancer Patients. Cancers 2019, 11, 1938. [CrossRef] [PubMed]

15. Ueda, M.; Iguchi, T.; Masuda, T.; Nakahara, Y.; Hirata, H.; Uchi, R.; Niida, A.; Momose, K.; Sakimura, S.; Chiba, K.; et al. Somatic mutations in plasma cell-free DNA are diagnostic markers for esophageal squamous cell carcinoma recurrence. Oncotarget 2016, 7, 62280-62291. [CrossRef] [PubMed]

16. Jay, J.J.; Brouwer, C. Lollipops in the Clinic: Information Dense Mutation Plots for Precision Medicine. PLoS ONE 2016, 11, e0160519. [CrossRef] [PubMed]

17. Zhou, Z.; Lyu, X.; Wu, J.; Yang, X.; Wu, S.; Zhou, J.; Gu, X.; Su, Z.; Chen, S. TSNAD: An integrated software for cancer somatic mutation and tumour-specific neoantigen detection. R. Soc. Open Sci. 2017, 4, 170050. [CrossRef]

18. Zhao, Y.; Wang, J.; Liang, F.; Liu, Y.; Wang, Q.; Zhang, H.; Jiang, M.; Zhang, Z.; Zhao, W.; Bao, Y.; et al. NucMap: A database of genome-wide nucleosome positioning map across species. Nucleic Acids Res. 2019, 47, D163-D169. [CrossRef]

19. Perdomo, S.; Avogbe, P.H.; Foll, M.; Abedi-Ardekani, B.; Facciolla, V.L.; Anantharaman, D.; Chopard, P.; Le Calvez-Kelm, F.; Vilensky, M.; Polesel, J.; et al. Circulating tumor DNA detection in head and neck cancer: Evaluation of two different detection approaches. Oncotarget 2017, 8, 72621-72632. [CrossRef]

20. Pittella-Silva, F.; Chin, Y.M.; Chan, H.T.; Nagayama, S.; Miyauchi, E.; Low, S.-K.; Nakamura, Y. Plasma or Serum: Which Is Preferable for Mutation Detection in Liquid Biopsy? Clin. Chem. 2020, 66, 946-957. [CrossRef]

21. Kubaczkova, V.; Vrabel, D.; Sedlarikova, L.; Besse, L.; Sevcikova, S. Cell-free DNA-Minimally invasive marker of hematological malignancies. Eur. J. Haematol. 2017, 99, 291-299. [CrossRef] [PubMed]

22. Wong, F.C.; Sun, K.; Jiang, P.; Cheng, Y.K.; Chan, K.A.; Leung, T.Y.; Chiu, R.W.; Lo, Y.M.D. Cell-free DNA in maternal plasma and serum: A comparison of quantity, quality and tissue origin using genomic and epigenomic approaches. Clin. Biochem. 2016, 49, 1379-1386. [CrossRef] [PubMed]

23. Cohen, J.D.; Li, L.; Wang, Y.; Thoburn, C.; Afsari, B.; Danilova, L.; Douville, C.; Javed, A.A.; Wong, F.; Mattox, A.; et al. Detection and localization of surgically resectable cancers with a multi-analyte blood test. Science 2018, 359, 926-930. [CrossRef]

24. Bouaoun, L.; Sonkin, D.; Ardin, M.; Hollstein, M.; Byrnes, G.; Zavadil, J.; Olivier, M. TP53Variations in Human Cancers: New Lessons from the IARC TP53 Database and Genomics Data. Hum. Mutat. 2016, 37, 865-876. [CrossRef] [PubMed]

25. Van der Meer, A.J.; Kroeze, A.; Hoogendijk, A.J.; Soussan, A.A.; van der Schoot, C.E.; Wuillemin, W.A.; Voermans, C.; van der Poll, T.; Zeerleder, S. Systemic inflammation induces release of cell-free DNA from hematopoietic and parenchymal cells in mice and humans. Blood Adv. 2019, 3, 724-728. [CrossRef]

26. Mansukhani, S.; Barber, L.J.; Kleftogiannis, D.; Moorcraft, S.Y.; Davidson, M.; Woolston, A.; Proszek, P.Z.; Griffiths, B.; Fenwick, K.; Herman, B.; et al. Ultra-Sensitive Mutation Detection and Genome-Wide DNA Copy Number Reconstruction by Error-Corrected Circulating Tumor DNA Sequencing. Clin. Chem. 2018, 64, 1626-1635. [CrossRef] [PubMed]

27. King, K.Y.; Huang, Y.; Nakada, D.; Goodell, M.A. Environmental influences on clonal hematopoiesis. Exp. Hematol. 2020, 83, 66-73. [CrossRef]

28. Delhomme, T.M.; Avogbe, P.H.; Gabriel, A.A.G.; Alcala, N.; Leblay, N.; Voegele, C.; Vallée, M.; Chopard, P.; Chabrier, A.; Abedi-Ardekani, B.; et al. Needlestack: An ultra-sensitive variant caller for multi-sample next generation sequencing data. NAR Genom. Bioinform. 2020, 2. [CrossRef] 
29. Fernandez-Cuesta, L.; Perdomo, S.; Avogbe, P.H.; Leblay, N.; Delhomme, T.M.; Gaborieau, V.; Abedi-Ardekani, B.; Chanudet, E.; Olivier, M.; Zaridze, D.; et al. Identification of Circulating Tumor DNA for the Early Detection of Small-cell Lung Cancer. EBioMedicine 2016, 10, 117-123. [CrossRef]

30. Martincorena, I.; Fowler, J.C.; Wabik, A.; Lawson, A.R.J.; Abascal, F.; Hall, M.W.J.; Cagan, A.; Murai, K.; Mahbubani, K.; Stratton, M.R.; et al. Somatic mutant clones colonize the human esophagus with age. Science 2018, 362, 911-917. [CrossRef]

31. Willenbrink, T.J.; Ruiz, E.S.; Cornejo, C.M.; Schmults, C.D.; Arron, S.T.; Jambusaria-Pahlajani, A. Field cancerization: Definition, epidemiology, risk factors, and outcomes. J. Am. Acad. Dermatol. 2020, 83, 709-717. [CrossRef]

32. Nordentoft, I.; Lamy, P.; Birkenkamp-Demtröder, K.; Shumansky, K.; Vang, S.; Hornshøj, H.; Juul, M.; Villesen, P.; Hedegaard, J.; Roth, A.; et al. Mutational Context and Diverse Clonal Development in Early and Late Bladder Cancer. Cell Rep. 2014, 7, 1649-1663. [CrossRef] [PubMed]

33. Grabuschnig, S.; Bronkhorst, A.J.; Holdenrieder, S.; Rodriguez, I.R.; Schliep, K.P.; Schwendenwein, D.; Ungerer, V.; Sensen, C.W. Putative Origins of Cell-Free DNA in Humans: A Review of Active and Passive Nucleic Acid Release Mechanisms. Int. J. Mol. Sci. 2020, 21, 8062. [CrossRef] [PubMed]

34. Fiala, C.; Diamandis, E.P. Utility of circulating tumor DNA in cancer diagnostics with emphasis on early detection. BMC Med. 2018, 16, 1-10. [CrossRef]

35. Islami, F.; Pourshams, A.; Nasrollahzadeh, D.; Kamangar, F.; Fahimi, S.; Shakeri, R.; Abedi-Ardekani, B.; Merat, S.; Vahedi, H.; Semnani, S.; et al. Tea drinking habits and oesophageal cancer in a high risk area in northern Iran: Population based case-control study. BMJ 2009, 338, b929. [CrossRef]

36. Aeberhard, W.H.; Cantoni, E.; Heritier, S. Robust inference in the negative binomial regression model with an application to falls data. Biometrics 2014, 70, 920-931. [CrossRef] [PubMed] 